\title{
POSTOPERATIVE AMYLOIDOSIS OF TONGUE BASE; EXTREMELY RARE COMPLICATION AFTER MULTILEVEL SLEEP SURGERY
}

\author{
Ahmed Bahgat ${ }^{1}$ and Claudio Vicini ${ }^{2}$ \\ ${ }^{1}$ Alexandria University \\ ${ }^{2}$ Osped Morgagni Pietrantoni
}

November 6, 2020

\begin{abstract}
This study presents a rare case of postoperative amyloidosis of the tongue base and provides a summary of the known literature of tongue amyloidosis. The diagnosis was by physical examination, radiological findings, and histopathological Congo red stain. Bone marrow biopsy the revealed "Plasma cell myeloma" as a cause.
\end{abstract}

Type of Manuscript: Case report.

Title:

Postoperative amyloidosis of tongue base; extremely rare complication AFTER multilevel sleep surgery

Short title: Tongue base amyloidosis

\section{Authors and Affiliations:}

Ahmed Bahgat ${ }^{(1)}$, MD; Claudio Vicini ${ }^{(2)}$, MD

(1) Department of Otorhinolaryngology, Alexandria university, Alexandria, Egypt

(2) Department of Head-Neck Surgery, Otolaryngology, Head-Neck and Oral Surgery Unit, Morgagni Pierantoni Hospital, Azienda USL della Romagna, Forlì, Italy

@ Corresponding author: Ahmed Bahgat, MD, Department of Otorhinolaryngology, Alexandria university, Elazaritta 00203 Alexandria, Egypt; Tel: +20-1141199933;

(email: ahmedyassinbahgat@gmail.com)

Keywords : Amyloidosis; tongue base surgery; Obstructive sleep apnea; Multilevel surgery, Postoperative complication.

Key Clinical Message : This is to put Amyloidosis in the differential diagnosis of postoperative edema of tongue base after tongue base ablation. After establishment of diagnosis of Amyloidosis, we have to exclude cause of secondary Amyloidosis

\section{Introduction}

Amyloidosis is a rare disorder where there is accumulation of pathologic deposits of amyloids in tissues. The amyloids are protein polymers formed of identical monomer units. Pathological amyloids are usually formed 
from misfolded proteins. The deposition of amyloids occurs either intracellularly or extracellularly alter the normal function of organs. ${ }^{(1)}$

Amyloidosis can be classified according to clinicopathological criteria as follows; i) primary systemic amyloidosis, with completely normal laboratory and radiological investigations; ii) secondary systemic amyloidosis caused by chronic disease, such as tuberculosis or rheumatoid arthritis; iii) Hereditary systemic amyloidosis associated with multiple myeloma; and iv) localized amyloidosis with no evidence of systemic amyloidosis or underlying chronic disease.

Head and neck amyloidosis can be either localized or part of systemic affection; Localized amyloidosis usually affect larynx and trachea. ${ }^{(2)}$ Tongue involvement is common in systemic amyloidosis either diffuse as macroglossia or localized. Localized tongue amyloidosis is extremely rare. ${ }^{(2)}$ This study presents a rare case of localized amyloidosis at the level of the tongue base following tongue base ablation surgery in the setting of multilevel surgery for management of severe obstructive sleep apnea patient.

\section{case report}

A 48-year-old male with a history of loud snoring and severe obstructive sleep apnea (OSA) as diagnosed by Polysomnography (Apnea/hypopnea index AHI of $58 / \mathrm{h}$ (normal $<5 / \mathrm{h}$ )). He was operated by septoplasty, inferior turbinectomy, tonsillectomy, UPPP, coblation tongue base ablation at one setting one year before presentation. The patient had a tough postoperative course, severe pain, edema of the neck and edema of tongue base. The patient suffered from Dysphagia, difficult breathing, change of voice and persistent snoring and OSA after surgery.

At presentation, the patient had diffuse submental and submandibular firm neck edema, limited mouth opening, firm edema of tongue base with limited tongue movement. (Fig. 1) A flexible endoscopy was performed, revealing some retropalatal adhesions, diffuse edema of the tongue base, adhesions of lateral border of tongue base with lateral oropharyngeal walls limiting full tongue movements, but otherwise normal upper aerodigestive tract with no obstructive lesions.

Radiological evaluation included Barium swallow but showed normal barium decent through the pharynx and esophagus. CT scan of neck showed only submental and submandibular edema with mural thickening of the pharyngeal walls. Those findings were confirmed by head and neck MRI with contrast, it revealed retropalatal adhesions, bilateral symmetrical swollen pterygoid muscles "pterygoid rhabdomyolysis" as described by radiologists, causing oro- \& hypo-pharyngeal luminal narrowing. (Fig. 2)

Drug induced sedation endoscopy (DISE) was performed to confirm diagnosis; and taking punch biopsy from tongue base \& FNAC from submental and submandibular edema tissue while patient is sedated. Biopsy revealed fibrous tissue with amorphous eosinophilic materials, no malignancy, no lymphoproliferative process. Further staining of the tissue with Congo red stain; Diagnosis was confirmed to be "Amyloidosis of tongue base".

Patient was referred to Hematologist to identify the ethology of Amyloidosis. Patient was investigated by complete lab work with immunological tests and bone marrow biopsy. Bone marrow biopsy revealed interstitial increase in plasma cells and lymphocytes, immunohistochemical staining (IHC) showed few scattered CD20 positivity with 50-60\% CD138 positive; A picture was suggestive of Plasma cell myeloma as a causative underlying etiology of amyloidosis. Patient started chemotherapy and steroids, he showed improvement in swallowing and less edema of the tongue, however, he was put on CPAP therapy for residual OSA.

\section{discussion}

Amyloidosis can be classified into systemic and localized, and can be classified according to the type of deposited fibrinogen into immunoglobulin light chain amyloidosis (AL), amyloid A amyloidosis (AA), $\beta 2$ microglobulin amyloidosis $(\mathrm{A} \beta 2 \mathrm{M})$ and transthyretin amyloidosis (ATTR). ${ }^{(3)}$ In systemic amyloidosis, the amyloid is deposited away from the site where it is produced, and it is transported via the circulatory system to the site of deposition. In localized amyloidosis, the location of amyloid production and deposition are the 
same. It is mainly caused by the AL amyloid, which is produced and deposited in local sites. ${ }^{(4)}$ The exact etiology of localized amyloidosis is not yet known.

When amyloidosis is suspected, it requires tissue biopsy under local anesthesia and microscopic examination (using H\&E and Congo red stains) are usually sufficient to establish a diagnosis. The next step is to exclude other organ involvements in systemic amyloidosis, then exclude underlying systemic disease (e.g. chronic inflammatory arthritis, tuberculosis, familial Mediterranean fever, Crohn's disease... etc). Finally, it's important to establish the subtype of amyloidosis; This is usually tested using serum or urine immunofixation electrophoresis to search for a clonal disorder. ${ }^{(5)}$

\section{Conclusion}

Recommendations regarding amyloidosis diagnosis are: i) Congo red stain is currently the gold standard for amyloid detection; ii) the type of amyloid must be identified microscopically or immunohistochemically, not solely on clinical or DNA studies. Cooperation with other medical specialties is crucial for correct and early diagnosis.

\section{Declarations:}

- Ethical approval and consent to participate: This study was approved by the ethics committee of Alexandria university. The participation in the study was approved by the patient.

- Consent for Publication: Taken from all authors and patient.

- This paper was presented as part of the International Surgical Sleep Society (ISSS) educational program.

- Availability of data and material: The datasets used and/or analysed during the current study available from the corresponding author on reasonable request.

- Authors Contribution: AB performed sleep endoscopy, tongue base biopsy, and FNAC from submental tissue edema. CV examined the patient and recommended doing biopsy, he was a major contributor in writing the manuscript. All authors read and approved the final manuscript.

- Acknowledgements: Many thanks to Prof. Claudio Vicini for his great experience.

- CONFLICT OF INTEREST: All authors declared no conflicts of interest.

- FINANCIAL DISCLOSURE: This study received no funding

\section{REFERENCES}

1. Buxbaum JN. The systemic amyloidoses. Curr Opin Rheumatol. 2004;16(1):67-75.

2. Musat G, Evsei A, Calina D, Docea AO, Doukas SG, Vageli DP, et al. Rare amyloidoma of the tongue base: A case report and review of the literature. Mol Clin Oncol. 2020;12(3):258-62.

3. Mollee P, Renaut P, Gottlieb D, Goodman H. How to diagnose amyloidosis. Intern Med J. 2014;44(1):7-17.

4. Pasternak S, White VA, Gascoyne RD, Perry SR, Johnson RL, Rootman J. Monoclonal origin of localised orbital amyloidosis detected by molecular analysis. Br J Ophthalmol. 1996;80(11):1013-7.

5. Deng J, Chen Q, Ji P, Zeng X, Jin X. Oral amyloidosis: A strategy to differentiate systemic amyloidosis involving the oral cavity and localized amyloidosis. Oral Dis. 2019;25(3):670-5.

\section{List of Figure legends}

Figure 1. Clinical presentation of the patient showing firm neck edema, firm tongue edema with limited mouth opening.

Figure 2. Radiological investigations of the patient; A. Barium swallow, and B. MRI of the head \& neck region. 

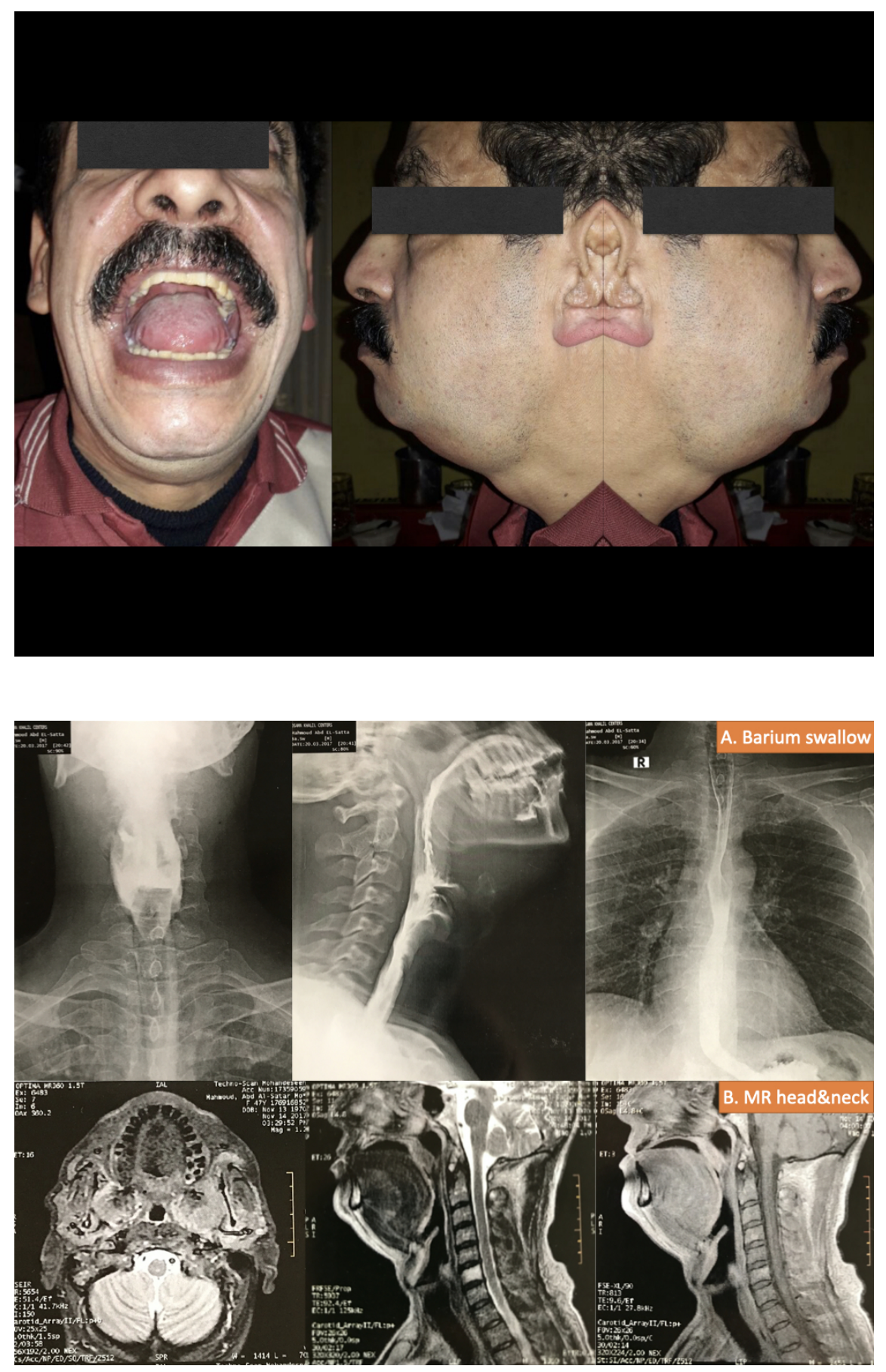